\title{
Isolation, characterization, antibiogram and pathology of Pasteurella multocida isolated from pigs
}

\author{
Mamta Tigga ${ }^{1}$, R. C. Ghosh ${ }^{2}$, Praveen Malik ${ }^{1}$, B. K. Choudhary ${ }^{3}$, Pratap Tigga ${ }^{2}$ and Dinesh Kumar Nagar ${ }^{1}$ \\ 1. National Research Centre on Equines, Sirsa Road, Hisar, Haryana, India; 2. Department of Veterinary Pathology, \\ College of Veterinary Science \& Animal Husbandry, Chhattisgarh Kamdhenu Veterinary University, Anjora, Durg, \\ Chhattisgarh, India; 3. ICAR Research Complex for Eastern Region, Patna, Bihar, India \\ Corresponding author: Mamta Tigga, email: chiyamum@gmail.com \\ Received: 26-03-2014, Revised: 22-04-2014, Accepted: 28-04-2014, Published online: 29-05-2014
}

doi: $10.14202 /$ vetworld.2014.363-368

How to cite this article: Tigga M, Ghosh RC, Malik P, Choudhary BK, Tigga P and Nagar DK (2014) Isolation, characterization, antibiogram and pathology of Pasteurella multocida isolated from pigs, Veterinary World 7(5): 363-368.

\begin{abstract}
Aim: Isolation, characterization and antibiogram of Pasteurella multocida from diseased pigs of district Durg of Chhattisgarh, and to study pathological changes caused by swine pasteurellosis.

Materials and Methods: An outbreak of swine pasteurellosis was suspected in pigs of Ruwabandha (Bhilai), Anjora, Somni, Tedesara, Tirgajhola villages of Durg district in Chhattisgarh, India during August and September of 2011. Nasal Swabs and blood samples from ailing pigs and heart blood and impression smears from morbid pigs were processed for detection and isolation of P. multocida by bacteriological methods. Detailed necropsy was conducted and gross and histopathological lesions were recorded. The test Isolates were subjected to antimicrobial sensitivity profile by disc-diffusion method.

Results: The blood smears from heart blood and tissue impression smears revealed teaming of bipolar organisms indicating the presence of Pasteurella spp. The isolates obtained were subjected to Gram's staining for checking the purity and bipolar morphology and characterized biochemically. Gross lesions included severe acute pneumonia and haemorrhages in lungs, petechial haemorrhages on serous membranes and other visceral organs. On histopathological examination, lungs showed typical fibrinous bronchopneumonia, multifocal suppuration. All the isolates of $P$. multocida were $100 \%$ sensitive to Amoxicillin, Gentamicin, Enrofloxacin and showed $100 \%$ resistance to Ceftizoxim and Cloxacillin.

Conclusion: Gross and microscopic lesions in dead animals are of great diagnostic value and are of characteristic of $P$. multocida infection. Cultural, morphological and biochemical characters are useful to rule out the causative agent as $P$. multocida. Antibiotic sensitivity pattern of the isolates should routinely be carried out for knowing the antibiotic resistance trends in an endemic area.
\end{abstract}

Keywords: Chhattisgarh, Pasteurella multocida, pathology, pneumonic pasteurellosis, swine pasteurellosis.

\section{Introduction}

Pasteurella multocida is a commensal and pathogen of respiratory tract of animals. Haemorrhagic Septicaemia is an acute infection of cattle, buffaloes, sheep and goats, caused by $P$. multocida capsular type $B$ and occasionally by $D$ and $E$. In swine, capsular types $\mathrm{A}$ and $\mathrm{D}$ are most often associated with atrophic rhinitis, while type A are associated with pneumonia, pleuritis and abscessation [1]. The disease occurs mainly in South East Asian countries and also in Africa, where it causes very heavy death losses. Haemorrhagic septicaemia is one of the most important bacterial diseases of cattle, buffalo and pigs in India. The disease occurs mainly during the rainy season. It spreads rapidly among groups of animals, causing morbidity and mortality between 50 to 100 percent. Pneumonic pasteurellosis is characterized by chronic pneumonia, purulent bronchopneumonia and pleurisy [2]. Affected pigs have fever of up to $106^{\circ} \mathrm{F}$, are anorectic and disinclined to move. They show significant respiratory

Copyright: The authors. This article is an open access article licensed under the terms of the Creative Commons Attribution License (http://creativecommons.org/licenses/by/2.0) which permits unrestricted use, distribution and reproduction in any medium, provided the work is properly cited. distress, often breathing through the mouth. Death is a common phenomenon after a clinical course of 4-7 days. There is a marked tendency for the disease to become chronic, resulting in reduced weight gains and frequent relapses. On post mortem examination there is a chronic bronchopneumonia with abscessation. Pleuritis is common and there may also be pericarditis. Peracute cases show an acute necrotizing fibrinous bronchopneumonia. Septicaemic disease with death occurring within 12 hours and without signs of pneumonia is sometimes observed in baby pigs. The disease occurs in all ages of pigs including adults and is manifested by fever, dyspnoea and congestion on serosal surfaces [3].

Chhattisgarh state of India is very rich in its livestock wealth with twelve million seven hundred thousand animals against twenty-five million five hundred thousand human population. Cattle population is the highest with $64 \%$, followed by goats (16\%), buffaloes $(14 \%)$ and sheep and pigs being the lowest (6\%). Livestock in Chhattisgarh are smaller in size with poor production potentialities, due to poor genetic potential coupled with inadequate availability of feed and fodder [4]. Pig production in Chhattisgarh is invariably a small-scale, backyard, marketed-oriented enterprise. It 


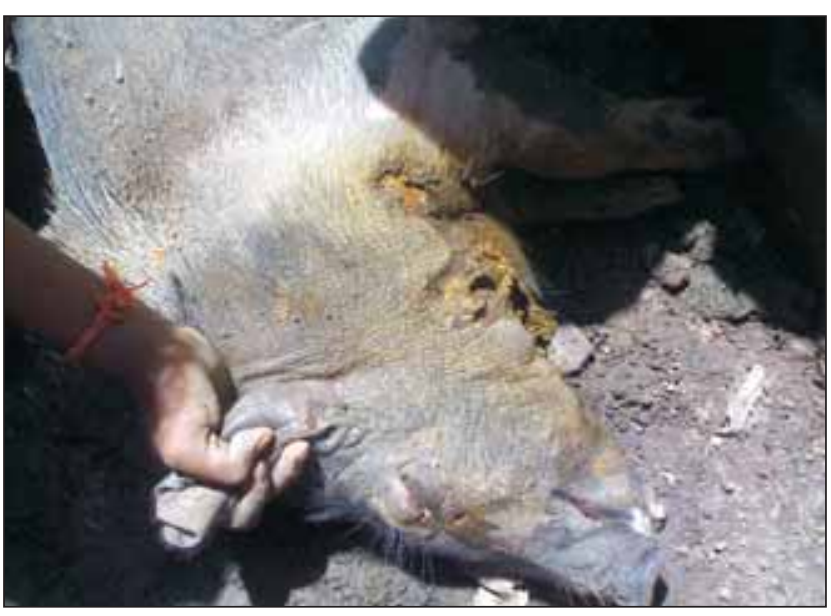

Figure-1: The photograph showing a case of oedematous swelling in the pharyngeal and ventral cervical region and brisket of adult Hampshire crossbred pig and subsequent scratch wound.

is practised mainly by Scheduled Tribes (ST) and some Other Backward Classes (OBC) to generate income, accumulate capital and fulfil socio-cultural obligations. Pigs are still considered scavenging animals and that the underprivileged are involved in pig production [5].

Swine Pasteurellosis and haemorrhagic septicaemia are controlled by vaccination of animals with H.S. vaccine. The vaccines preparation used for cattle buffaloes and pigs are same. The vaccinated and non-vaccinated animals were affected during each outbreak of swine pasteurellosis in Chhattisgarh with variable mortality rates and different disease manifestations. It indicates that there is possibility of variation in antigenicity between vaccine strain and field isolate of Pasteurella. Therefore, there is urgent need of bacteriological and pathological characterization the potential pathogen $P$. multocida involved in recurrent outbreaks.

\section{Materials and Methods}

Case description: An outbreak of swine pasteurellosis was suspected in pigs of Ruwabandha (Bhilai), Anjora, Somni, Tedesara, Tirgajhola villages of Durg district in Chhattisgarh state of India during August to September of 2011. The villages are located within 30 kilometres of radius. Altogether, there were around 50 pig rearing families having 5-30 pigs per family. A total number of 377 pigs were under risk of the disease, out of which 249 animals were died. A total number of 126 animals were attended for necropsy and the representative tissue samples were collected for histopathological and bacteriological examination. The overall mortality rate was $66 \%$. The affected animals were off fed and had high fever $\left(41-42^{\circ} \mathrm{C}\right)$. The atmospheric temperature and humidity were recorded between $24.5^{\circ} \mathrm{C}-29.5^{\circ} \mathrm{C}$ and $76 \%-93 \%$ respectively. Socio-economically poor families used to rear pigs under backyard system and housed in poorly managed muddy houses. These animals were fed with agricultural and kitchen waste and were allowed to graze in fields. Pig farmers had little knowledge of pig diseases and health care management. Outbreak of disease caused high mortality within all age group of

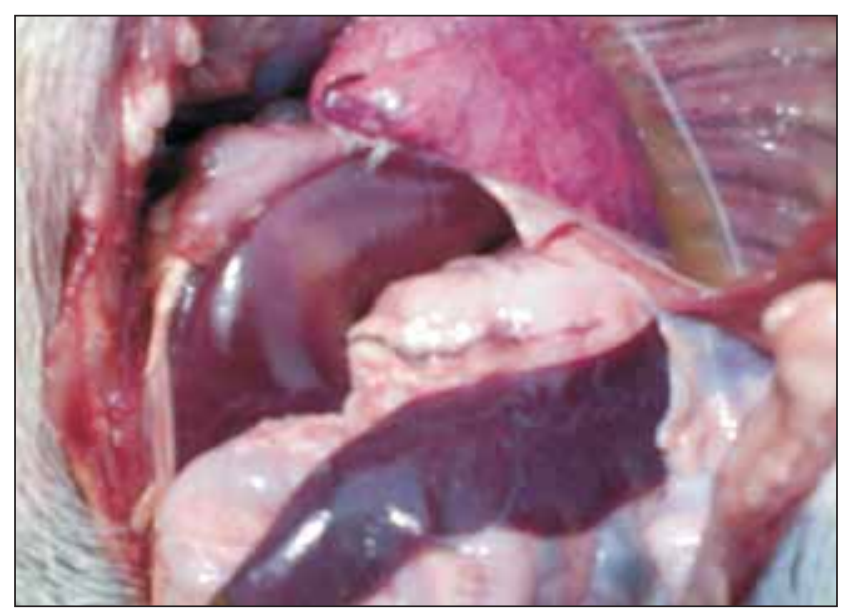

Figure-2: The photograph showing consolidation of lungs, hydrothorax, congested liver and spleenomegaly of pig died of swine pasteurellosis

animals. Clinical signs exhibited by ailing adult pigs were pyrexia $\left(41-42^{\circ} \mathrm{C}\right)$, staggering gait, dullness, serous nasal discharge and dyspnoea leading to death after a clinical course of 4-6 days. Case fatality rate was $95 \%$ in adult animals and $100 \%$ in piglets. Infected piglets showed high fever and serous nasal discharge and died within 24 hours of onset of fever. A very few pigs were treated with Enrofloxacin. Atypical cases of oedematous swellings noted in the pharyngeal region, these swellings spread to the ventral cervical region and brisket of adult Hampshire crossbred pigs. Later, the edematous part was scratched by affected pigs and ulcerated wound followed (Figure-1).

\section{Processing of samples}

Pathological Studies: The infected pigs were examined clinically. Detailed necropsy was conducted and gross lesions were recorded. Tissue samples from heart, lungs, liver, spleen, kidneys and Lymph nodes were collected in 10\% Formal saline solution for histopathological studies. Morbid tissue samples then processed by routine histopathological techniques and stained with haematoxylin and eosin stains [6].

Bacteriological Isolation: The blood smears were prepared from heart blood and the impression smears were prepared from Lungs, spleen and stained with Methylene Blue and Leishman's stain to demonstrate the causative organisms. Heart blood, nasal swabs and tissue samples from lungs and spleen were collected and processed for bacteriological isolation. The clinical samples for bacteriological examination were collected by using sterile HiMedia Transport swabs (HiMedia Laboratories Pvt. Ltd., Mumbai, India).

For primary isolation of the organism the samples were inoculated on Sheep Blood agar (SBA) and Mac Conkey agar (MLA) according to methods described by Cruickshank et al. [7] and Quinn et al. [2] The pure cultures were transferred to Brain Heart Infusion (BHI) Agar slants for further identification. P.multocida isolates were identified by biochemical tests viz., Oxidase, Catalase, Indole, Citrate utilization, Nitrate reduction 
Table-1: Showing the antibiotic sensitivity pattern of P. multocida

\begin{tabular}{lccc}
\hline Antibiotic & \% of sensitive isolates & \% of resistant isolates & \%e of intermediate isolates \\
\hline Ceftizoxim(Ck) & 0 & 100 & 0 \\
Amoxycillin (Am) & 100 & 100 & 0 \\
Cloxacillin (Cx) & 0 & 95.6 & 0 \\
Roxithromycin (Ro) & 4.3 & 0 & 0 \\
Oxacillin (Ox) & 01.3 & 0 & 8 \\
Amikacin (Ak) & 100 & 100 & 0 \\
Gentamicin (G) & 0 & 0 & 0 \\
Cephalexin (Cp) & 100 & 0 & 0 \\
Trimethoprim (Tr) & 78.2 & 0 & 0 \\
Cotrimoxazole (CoT) & 100 & 0 & 0 \\
Oxytetracycline (O) & 100 & 0 & 0 \\
Enrofloxacin (Ex) & 100 & 0 & 0 \\
Chloramphenicol (C) & 100 & 0 & 0 \\
Sulphadiazine (Sz) & 100 & 0 & 0
\end{tabular}

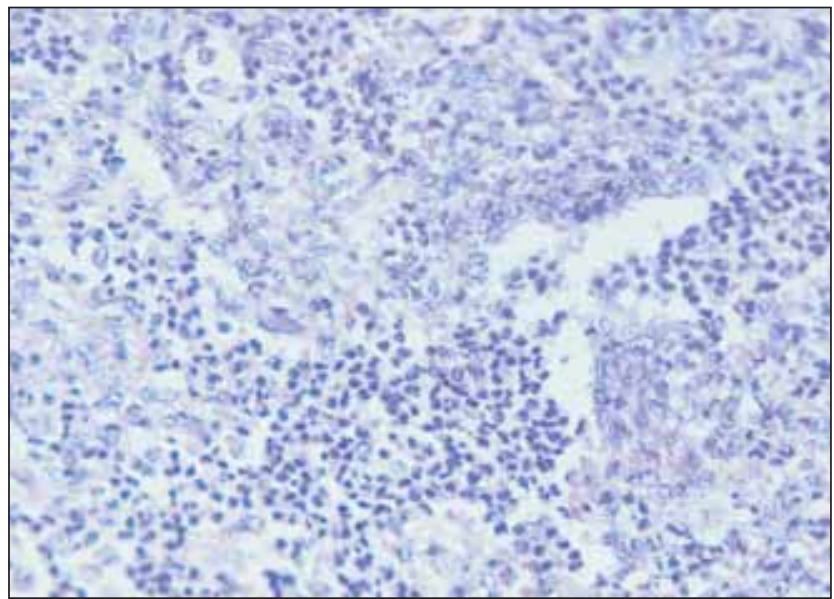

Figure-3: Microphotograph showing fibrinous exudate and flooding of polymorphonuclear cells in alveoli.

and fermentation of sugars viz., glucose, mannitol, sucrose, mannose, maltose, arabinose, lactose, dulcitol, salicin, inositol and trehalose as per methods described by Cowan and Steel [8].

Antimicrobial drugs sensitivity pattern of $P$. multocida: All the isolates were tested for sensitivity pattern against 15 different antimicrobial agents (Table-1) lcommonly used in field practices. Antibiotic discs from HiMedia Laboratories Pvt Limited, Mumbai (India) were used for in-vitro antibiotic sensitivity test as per the method described by Bauer et al. [9]. The diameters of inhibition zones surrounding the antibiotic discs were measured and subsequently matched with the standard inhibition zone diameters of respective antibiotic discs. On the basis of size of inhibition zones of various antibiotics, the isolates were classified as sensitive, intermediately sensitive or resistant.

\section{Results}

Gross and histopathology: On post-mortem examination, Petechiae and frothy exudate were present in trachea and bronchi. Petechiae were seen all over the serous membranes and peritoneum. There was hydrothorax with accumulation of straw coloured fluid. Endocarditis was observed in the heart. Lungs showed severe consolidation along with the presence of multiple focal haemorrhages. Spleens from most of the dead pigs were very much enlarged (Figure-2).

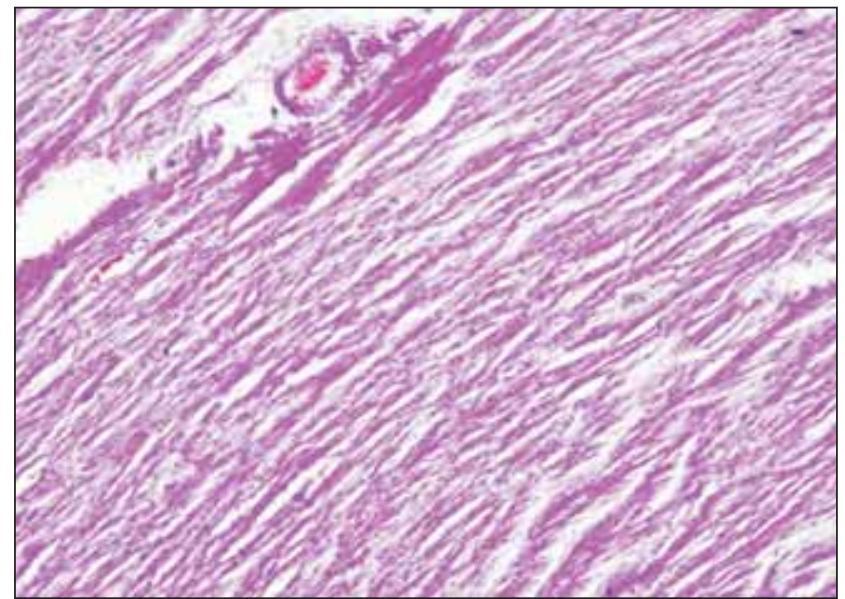

Figure-4: Microphotograph showing presence of thrombi in blood vessel of heart

Liver and kidneys were swollen and contained petechial haemorrhages. Lymph nodes were enlarged, oedematous and haemorrhagic.

Microscopically, lungs showed typical fibrinous bronchopneumonia, multifocal suppuration, septae were thickened with fibrin, combined with cellular infiltration and edema. Alveoli were filled with fibrinous exudate, erythrocytes and polymorphonuclear cells (Figure-3). Pleura were severely thickened. There were sub-pleural haemorrahages. Heart of some pigs showed presence of thrombi, haemorrhages and necrosis of myocardium (Figure-4). Glomeruli and kidney tubules showed haemorrhages and necrotic changes. Severe congestion and haemorrhages were observed both in cortex and medulla of lymphnodes. There were haemorrhages and necrosis in Liver.

Isolation and characterization of $\boldsymbol{P}$. multocida: The blood smears from heart blood and tissue impressions revealed teaming number of bipolar organisms indicating the presence of Pasteurella spp. The organisms were isolated from morbid tissue samples viz. lungs, heart blood and spleen as well as clinical blood/nasal samples of the pigs. Clinical samples, on sheep blood agar plates yielded tiny transparent, non-hemotytic colonies after an incubation of 24 hours at $37^{\circ} \mathrm{C}$ (Figure-5a). Subsequently, the non-haemolytic single colony from SBA was transferred to MLA plate and incubated at $37^{\circ} \mathrm{C}$ for 24 hours. The isolates which 


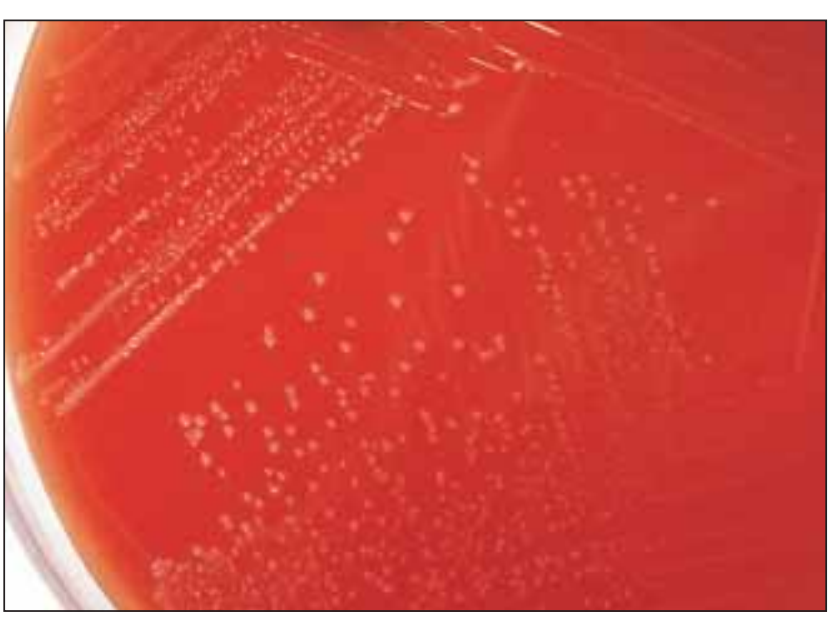

Figure-5a: Photograph showing non-hemolytic smooth colonies of $P$. multocida on SBA plate.

failed to grow on MLA were preliminary presumed to be $P$. multocida. Single non-haemolytic colony of these isolates was picked up from primary culture and restreaked on fresh SBA plate and incubated at $37^{\circ} \mathrm{C}$ for 24 hours to obtain single colony of pure culture of the isolates. On the basis of Gram staining, the isolates were Gram's negative, cocco-bacillary rods (Figure$5 b$ ). The isolates so obtained were confirmed by biochemical characterization. In the present study, the test isolates were found to be positive for oxidase, catalase, indole production and reduction of nitrate. The test isolates fermented dextrose, trehalose, xylose and mannitol. The isolates failed to ferment lactose, arabinose and dulcitol.

Antimicrobial sensitivity test: The test isolates were found $100 \%$ sensitive to Amoxycillin, Gentamicin, Enrofloxacin, Sulphadiazine, Ofloxacin, Trimethoprim and Amikacin (91.3\%), Cotrimoxazole (78.2\%). Whereas, the isolates showed $100 \%$ resistance against Ceftizoxim, Cephalexin, Cloxacillin and 95.6\% resistance was shown against Roxithromycin. The results of in vitro antibiotic sensitivity were interpreted and are depicted in Table-1.

\section{Discussions}

P. multocida is considered a commensal organism in the upper respiratory tract and tonsils and causes disease outbreaks in swine, cattle, buffalo, sheep, and goats under extreme environmental conditions. This organism is the most common pathogen isolated from pigs housed under poor husbandry conditions, eg, overcrowding and poor ventilation. Source of infection in recent outbreak was introduction of a sick pig from slaughter market of Bhilai. In the present study, case fatality rate was $95 \%$ in adult pigs and $100 \%$ in piglets. The high mortalities observed in many outbreaks of pasteurellosis in Chhattisgarh and other parts of India [2, 10-13]. Similar outbreak of swine pasteurelosis in pig herd has been reported earlier with high mortality and variable degree of ulcerative skin lesions $[11,14]$. The ulcerative skin lesions were absent in pigs from recent outbreak but oedematous swellings were noted

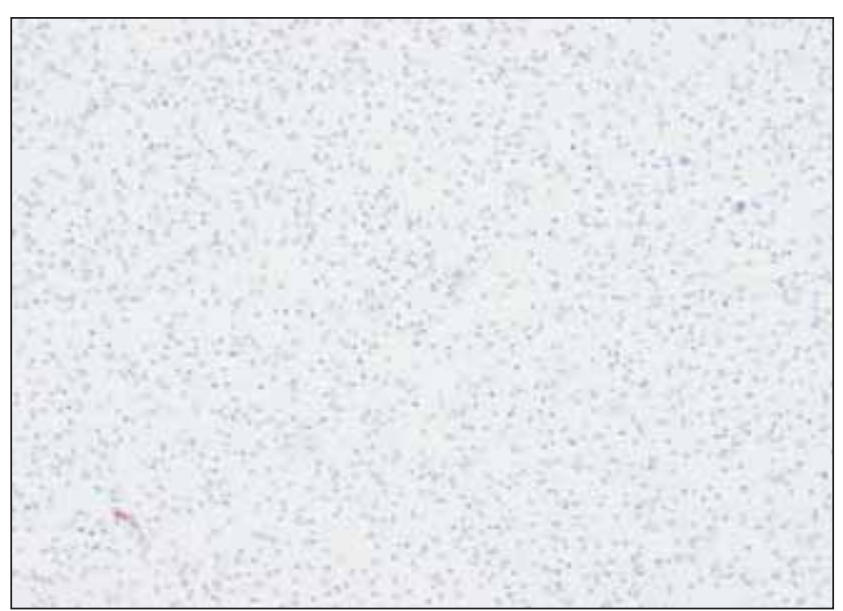

Figure-5b: Microphotograph showing $P$. multocida as coccobacillary bipolar rods.

in the pharyngeal region of some of the infected pigs which is the predominant manifestation in cattle and buffaloes. The oedematous swellings in the pharyngeal region of diseased pigs were unique finding during this study. P multocida type B:2, is responsible for haemorrhagic septicemia in dairy cattle and buffalo and many outbreaks are reported every year from all over India, suggesting that this serotype may have transmitted between bovine species and swine during recent outbreak $[11,12,15]$. The outbreak of pasteurellosis is attributed to impairment of host defences mechanism, strain and virulence of causative organism and various other physiological and environmental stress factors [8]. Thus, due to highly contagious nature of the disease and high mortality rate, the disease has played a major role in huge economic loss in rural population of Chhattisgarh.

Post-mortem findings, pathological changes and demonstration of bipolar organisms in impression smears suggestive of swine pasteurellosis have also been reported by other workers $[11,13,16]$. Bronchopneumonia with abscessation and pleuritis have been found to be common lesions of pneumonic pasteurellosis in pigs $[2,11,14]$. Fibrinous and suppurative bronchopneumonia with focal areas of necrosis typical of pneumonic pasteurellosis was found. Similar lesion were recorded by Parveena et al. [16].

On blood agar plates, the colonies of $P$. multocida isolate were non- hemolytic, round, smooth or mucoid. The isolate failed to grow on MacConkey's agar. On the basis of Gram's staining, the isolates were found to be Gram negative and cocco-bacillary rods in morphology. The test isolates of $P$. multocida organisms were confirmed by biochemical characters. These findings are similar to the findings of Sharma et al. [17], Kalorey et al. [12], Quinn et al. [2], and Jogi [14]. In the present study, the isolates were found to be positive for oxidase, catalase, indole production and reduction of nitrate. The test isolates fermented glucose and sorbitol but failed to ferment lactose, arabinose and adonitol. This is in accordance with the finding of Verma [18] and Jogi [13]. Rajni et al. [19] recorded that most of the 
avian isolates ferment arabinose while mammalian isolates failed to ferment arabinose. The variability observed in fermentation reactions of carbohydrates might be due to geographical variation of isolates and use of chemotherapeutic agents, as these factors influence the enzyme profiles of microbes. $[13,19]$.

The majority of test isolates were found to be $100 \%$ sensitive to Amoxycillin, Gentamicin, Enrofloxacin, Sulphadiazine, Ofloxacin, Trimethoprim, Chloramphenicol and Oxytetracycline. Higher efficacy of enrofloxacin and ofloxacin along with chloramphenicol has also been reported by Sharma et al. [15] and Varte et al. [14]. Enrofloxacin and chloramphenicol were found to be quite effective against pasteurellosis by several research groups $[14,20]$. The isolates in this study were $100 \%$ resistant to Cephalexin. Balakrishnan et al. [21] recorded that $P$. multocida were resistant to cephalexin. Varte et al. [15] and Jogi [14] recorded $100 \%$ sensitivity for Cephalexin. Rajkhowa et al. [22] recorded the $P$. multocida isolates from pigs were resistant to Cephalexin and Sulphadiazine. The resistance for Cephalexin in the present study could be due to development of resistance towards Cephalexin. Thus, it shows the necessity of in vitro antibiotic sensitivity prior to treatment.

\section{Conclusion}

There was an outbreak of swine pasteurellosis in pigs of different villages in Chhattisgarh with a case fatality rate of $90 \%$ in adult pigs and $100 \%$ in piglets. Although there are recurrent outbreaks of swine pasteurellosis in Chhattisgarh state but the clinical manifestations varies in each outbreak. It is observed in last one decade that there is a shift in antibiotic sensitivity pattern of $P$. multocia. The isolates observed resistance against conventional antibiotics particularly to Cephalexin, The same drug was found to be $100 \%$ sensitive during previous outbreaks which are leading to problems in treating the affected animals. This warrants the need for the thorough clinical and microbiological investigation of $P$. multocida in each outbreak. Furthermore, the results of this study emphasize the importance of in vitro antibiotic sensitivity test prior to initiate treatment.

\section{Authors' contributions}

MT: Collection of Samples and processing for isolation, characterization and antibiotic sensitivity test of $P$. multocida. Histopathological examination and manuscript writing. RCG: Planning of research methodology, technical support, histopathological examination and editing of manuscript. PM: Planning of research methodology, technical support and editing of the manuscript. All authors read and approved the final manuscript. BKC and PT: Collection and processing of samples for bacteriology and histopathology. DKN: Processing of samples for biochemical and molecular characterization. All authors read and approved the final manuscript.

\section{Acknowledgements}

The authors are thankful to the Dean, Veterinary College, Anjora, Durg, Chhattisgarh and Director, National Research Centre on Equines, Hisar, Haryana for providing the necessary facilities to carry out the research. Preliminary isolation of bacteria and histopathology work were done at Veterinary College, Anjora, Durg, Chhattisgarh and further charcterization of isolates and their antibiogram were done at Veterinary Type Culture Centre (VTCC), NRCE Hisar. VTCC is a unit under NRCE which is involved in collection, characterization, preservation and accession of pathogens of animal origin. Fund for the study was provided by Indian Council of Agricultural Research and Veterinary College, Anjora, Durg, Chhattisgarh, India.

\section{Competing interests}

The authors declare that they have no competing interests.

\section{References}

1. Zhao, Guosong, Thomas, Halbur, Duane, C. And Pankratz (1995) Colonization of oropharynx and nasal cavity of CDCD pigs by a nontoxigenic strain of Pasteurella multocicda type D. J. Swine Health Prod. 3(3):113-115.

2. Quinn, P.J., Carter, M.E, Markey, B.K., and Carter, G.R. (1994) Veterinary Clinical Microbiology. Wolfe Publication, London, U.K. p254-258.

3. Murty, D.K., Kaushik, R.K. (1965) Studies on an outbreak of acute swine pasteurellosis due to Type B. Vet. Rec. 77: 411416.

4. Final Third RFP for ILDP FSDC 2012-13 RKVY. http://ahd.cg.gov.in/PDF_common/Final\%20Third\%20RF P\%20for\%20ILDP\%20F Accessed on 21/2/2014.

5. Rangnekar, D.V. (2006) Livestock in the livelihoods of the underprivileged communities in India: A Review. International Livestock Research Institute, Nairobi, Kenya.p32,45. http://www.ilri.org/Infoserv/webpub/fulldocs/Livestock InTheLivelihoods/Livestock UP_India_Final.pdf. Accessed on $21 / 2 / 2014$.

6. Jubb, K.V.F., Kennedy, P.C. and Palmer N. (1993) Pathology of Domestic Animals. 4th edn. Academic press, Vol.2, London, p447-522.

7. Cruickshank, R., Duguid, J.P., Marimon, B.P. and Swain, R.A.H. (1975) Medical Microbiology. 2nd Edn. Vol. I, Church Hill, Livingstone, Edinburgh. p369-470.

8. Cowan, S.T. and Steel, K.J. (1965) Manual for the identification of medical bacteria. Cambridge University Press.p127.

9. Bauer, A.W.; Kirby, W.M.M.; Sherris, J.C. and Turck, M. (1966) Antibiotic susceptibility testing using a standard single disc method. Am. J. Clin. Pathol. 45:493-496.

10. Prabhakar, T.G., Prabhakar, P. and Venkadabadi (2010) Molecular characterization of Pasteurella multocida isolated from an incidence of sheep pasteurellosis in Karamadai hill tract of Tamilnadu. TNJVAS., 6(2) 81-87.

11. Ghosh, R.C., Hirpurkar, S.D. and Mondal, M. (2011). An outbreak of swine pasteurellosis in Chhattisgarh. Indian J. Vet. Pathol. 35 (1):87-88.

12. Kalorey, D.R., Yuvaraj, S., Vanjari, S.S., Gunjal, P.S., Dhanawade, N.B., Barbuddhe, S.B. and Bhandarkar, A.G. (2008) PCR analysis of Pasteurella multocida isolates from an outbreak of pasteurellosis in Indian pigs. Comp. Immun. Microbiol. Infect. Dis. 31:459-465.

13. Mohamed-Wael, Mohamed A., Moemen A. Mohamed (2014) Molecular analysis of Pasteurella multocida strains isolated from fowl cholera infection in backyard chickens. Asian Pac. J. Trop. Biomed. 4(1): 8-12. 
14. Jogi, J. (2006) The microbial architecture of Pasteurella multocida isolated from swine. M.V.Sc. Thesis submitted to Indira Gandhi Krishi Vishvavidylaya, Raipur, India.

15. Varte Z, Dutta TK, Roychoudhury P, Begum J and Chandra R (2014) Isolation, identification, characterization and antibiogram of Pasteurella multocida isolated from pigs in Mizoram with special reference to progressive atrophic rhinitis, Vet. World 7(2): 95-99.

16. Praveena,P. E., Periasamy, S., Kumar, A. A. and Singh N. (2014) Pathology of Experimental Infection byPasteurella Multocida Serotype A1 in Buffalo Calves. Vet. Pathol. doi:10.1177/0300985813516647 (give vol and page no. if available at the site).

17. Sharma, S.P., Sharma, R.K. and Rahman, H. (2005) Isolation, serotyping and antibiogram of Pasteurella from pigs of North-East India. Indian. J. Anim. Sci., 74: 905-907.

18. Verma, N.D., (1991) Type B:2 P. multocida in an outbreak of primary swine pasteurellosis Indian J. Anim. Sci. 61:158160.

19. Rajini, R.; Sesnagiri, R.A.; Dhanalakshmi, K and Sharma, B.J.R. (1995) Studies on avian pasteurellosis in Andhra Pradesh. Indian Vet. J. 72:115-118.

20. Sellyei, B., Varga, Z., Szentesi, S.K., Kaszanyitzky, E. And Magyar, T. (2009) Antimicrobial susceptibility of Pasteurella multocida isolated from swine and poultry. Acta Vet. Hung. 57(3):357-67.

21. Balakrishnan G, Roy, P, Nagarajan K, Selvaraj, J., Manohar, B.M. (2012) Isolation, identification and antibiogram of Pasteurella multocida isolates of rabbits suffering from pasteurellosis. IJAVMS., 6(1): 58-61.

22. Rajkhowa, S., Shakuntala, I., Pegu, S.R., Das, R.K. and Das, A. (2012) Detection of Pasteurella multocida isolates from local pigs of India by polymerase chain reaction and their antibiogram. Trop. Anim. Health Prod. 44(7):1497-1503.

$* * * * * * * *$ 